\title{
ÖKONOMISCHE ÜBERLEGUNGEN ZUM WISSENSCHAFTSBETRIEB: IMMATERIALGÜTERRECHTE, WISSENSALLMENDE UND INFORMATIONSNACHHALTIGKEIT
}

\begin{abstract}
Nachhaltigkeit und nachhaltiges Wirtschaften sind spätestens seit Mitte der 1980er Jahre wichtige Konzepte sowohl wissenschaftlicher als auch politischer Debatten. Sie werden insbesondere thematisiert, wenn es um den Umgang mit knappen materiellen Ressourcen geht - fossile Energieträger sind hierfür sowohl als endliche Ressourcen als auch als Gefährdung anderer knapper Ressourcen wie eine saubere Umwelt ein paradigmatisches Beispiel. Da es jedoch nicht nur materielle Ressourcen, sondern auch Immaterialgüter gibt, soll untersucht werden, ob Konzepte der Nachhaltigkeit und des nachhaltigen Wirtschaftens auch in Bezug auf Immaterialgüter wie Informationen und Wissen angewendet werden können. Dazu wird es notwendig sein, zunächst die spezifischen ökonomischen Eigenschaften von Informationen und Wissen herauszuarbeiten. Dabei zeigt sich, dass Immaterialgüter durch Anwendung von Immaterialgüterrechten zu knappen Ressourcen gemacht werden können. Wenn aber auch Informationen und Wissen faktisch zu den knappen Ressourcen gezählt werden müssen, so macht es Sinn, Nachhaltigkeit auch für Immaterialgüter zu fordern - dies soll mit dem Ausdruck „Informationsnachhaltigkeit“ bezeichnet werden. Wird das Konzept der Informationsnachhaltigkeit auf den Wissenschaftsbetrieb angewendet, so kann in Bezug auf den Wissensbestand der Wissenschaft von einer Wissensallmende gesprochen werden. Die schon länger stattfindenden Veränderungen des Wissenschaftsbetriebs und des wissenschaftlichen Publikationswesens gefährden auf der einen Seite den Bestand der Wissensallmende; auf der anderen Seite finden sich jedoch auch Gegenreaktionen, die Versuche darstellen, bspw. durch den Open Access-Gedanken die Wissensallmende zu erhalten. Allerdings zeigt die Analyse, dass sowohl eine romantisierende Perspektive auf die Wissensallmende und Open Access als auch eine pauschale Kritik am existierenden privatwirtschaftlichen Publikationswesen falsch wären, da beide Teile des wissenschaftlichen Publikationswesens aufeinander angewiesen sind.

Schlüsselbegriffe: Nachhaltigkeitsforschung, Ressourcenmanagement, Immaterialgüter, Informationsnachhaltigkeit, Wissenschaftsökonomik.
\end{abstract}

\section{VORBEMERKUNGEN}

Im Kontext des Umweltschutzes ist Nachhaltigkeit spätestens seit Mitte der 1980er Jahre ein wichtiges Konzept. Zunächst vor allem in Expertenkreisen diskutiert, wird Nachhaltigkeit heute auch in öffentlichen Debatten angesprochen, vor allem seit deutlich wird, dass fossile Energieträger zum einen endlich sind und zum anderen deren Verbrauch zu massiven Klimaänderungen beitragen kann. Allgemein gesprochen werden Nachhal-

${ }^{1}$ Prof. Dr Karsten Weber, Lehrstuhl für Allgemeine Technikwissenschaften, BTU Cottbus, Postfach 101344; 03013 Cottbus, tel. 49 (0) 355 69-0, e-mail: karsten.weber@ b-tu.de 
tigkeit und nachhaltiges Wirtschaften dann thematisiert, wenn es um den Umgang mit knappen materiellen Ressourcen geht. Doch der Mensch lebt nicht nur vom Brot allein; nicht alles, was Menschen benötigen, ist materieller Natur: Für moderne Volkswirtschaften ist der Zugang zu Informationen und Wissen von essentieller Bedeutung, ebenso wie für Individuen bspw. im Kontext von Bildung. Wenn Informationen und Wissen also wichtige Güter sind, stellt sich die Frage, ob sie ebenfalls nachhaltig bewirtschaftet werden müssen, damit die Probleme und Konflikte im Umgang mit materiellen Gütern nicht auch in der Sphäre immaterieller Güter auftauchen.

Übliche Beispiele für Nachhaltigkeit beziehen sich in der Regel auf einen knappen Rohstoff; Nachhaltigkeit bedeutet dann unter anderem, diesen Rohstoff durch Recycling so verlustfrei wie möglich einzusetzen oder, wo immer möglich, einen nicht nachwachsenden Rohstoff durch einen nachwachsenden zu ersetzen. Bezüglich der Energieversorgung wird von Nachhaltigkeit gesprochen, wenn Energiequellen im Rahmen menschlichen Handelns nicht erschöpfbar sind, so bspw. Windenergie, Solarthermie und Photovoltaik, Geothermie, Wasserkraft oder nachwachsende Biotreibstoffe. Der sparsame oder nachhaltige Gebrauch von Rohstoffen wurde zunächst in Bezug auf den Wirtschaftskreislauf und den Schutz der Umwelt diskutiert, doch wird inzwischen auch von sozialer Nachhaltigkeit gesprochen: Soziale Verhältnisse einer Gesellschaft sind dann nachhaltig, wenn sie soziale Stabilität, allgemeinen Wohlstand und das friedliche, kooperative und gleichberechtigte Zusammenleben der Bürgerinnen und Bürger einer Gesellschaft fördern.

Wissen und Informationen können nicht auf die gleiche Weise verbraucht werden wie materielle Güter. Sie sind, in der Sprache der Ökonomie, reine öffentliche Güter, da ihre Nutzung, zumindest prima facie, nicht rival ist und niemand von vornherein von der Nutzung exkludiert wird - erst die Anwendung von Immaterialgüterrechten lässt Informationsgüter zu einem knappen Gut werden. Da Wissen und Informationen andere Eigenschaften als materielle Güter besitzen, sollen daher zunächst diese Eigenschaften identifiziert, der Nachhaltigkeitsbegriff bezüglich Wissen und Informationen näher bestimmt und eine Antwort darauf gegeben werden, ob die so definierte Informationsnachhaltigkeit ${ }^{2}$ einen Beitrag zur Erhaltung der globalen Wissensallmende leisten kann. Zusätzlich sollen Aufgaben(felder) skizziert werden, die mit der Idee von Informationsnachhaltigkeit und Wissensallmende verbunden sind. Viele dieser Aufgaben sind nicht neu, sondern stellten sich bspw. Bibliotheken und Archiven bereits in der Vergangenheit; angesichts der digitalen Revolution erscheint deren Lösung nun jedoch noch drängender.

${ }^{2}$ In diesem Text wird unter anderem das Konzept der Informationsnachhaltigkeit behandelt; dieser Ausdruck ist bisher allerdings noch nicht etabliert. Rainer Kuhlen (2004: 31), der sich in Deutschland sehr für den freien Zugang zu Wissen stark gemacht hat, spricht bspw. von einer ,wissensökologischen Perspektive“: „Hierbei wird die Idee der Nachhaltigkeit nicht mehr allein auf die natürlichen Ressourcen, sondern auch auf die intellektuellen Ressourcen bzw. auf den Umgang mit Wissen und Information bezogen [...]. Dazu muss das bisherige Drei-Säulen-Modell der (sozialen, ökonomischen und ökologischen) Nachhaltigkeit um die informationelle Dimension erweitert werden." Inhaltlich ist dieser Gedanke also sehr nahe bei dem, was auf den folgenden Seiten beschrieben wird. 


\section{INFORMATION, INFORMATIONSGESELLSCHAFT,} INFORMATIONSNACHHALTIGKEIT

Die Situation der Menschen auf unserem Planeten ist durch die Gleichzeitigkeit großer Unterschiede und Ungleichheiten geprägt; die Spanne von kaum mehr vorstellbarem Reichtum auf der einen und erbarmungswürdiger und ebenso schwer vorstellbarer Armut auf der anderen Seite ist nur ein Aspekt dieser Unterschiede und Ungleichheiten. Ein anderer Aspekt sind die Möglichkeiten der Kommunikation und des Zugangs zu Informationen und Wissen. Zwar nutzen inzwischen weit über zwei Milliarden Menschen das Internet, doch bei einer Weltbevölkerung von mehr als sieben Milliarden Menschen heißt das, dass wenigstens zwei Drittel aller Menschen von der Nutzung des Internet ausgeschlossen und von den Vorteilen des Lebens in einer Informationsgesellschaft weit entfernt sind (Castells 2001: 247):

„The differentiation between Internet-haves and have-nots adds a fundamental cleavage to existing sources of inequality and social exclusion in a complex interaction that appears to increase the gap between the promise of the Information Age and its bleak reality for many people around the world.“

Was eine Gesellschaft als „Informationsgesellschaft“ kennzeichnet, ist nicht völlig klar (vgl. Bittlingmayer \& Bauer 2004: 50; Gault \& McDaniel 2002; Rai \& Lal 2000); um dies feststellen zu können, wären objektive und messbare Indikatoren notwendig, die die Transformation hin zu einer Informationsgesellschaft anzeigten (vgl. Britz 1999: 9; Iljon 1996: 11f.). Solche Indikatoren könnten bspw. messen, welchen Stellenwert Informationen $^{3}$ in den verschiedenen Bereichen gesellschaftlichen und individuellen Lebens besitzen. Informationsgesellschaften zeichnen sich im Vergleich zu anderen Gesellschaftstypen vermutlich dadurch aus, dass in beinahe allen Bereichen des politischen, öffentlichen und privaten Lebens Informationen sehr wichtige, wenn nicht gar die wichtigsten Faktoren für Entscheidungsfindungs- und Problemlösungsprozesse sind; sie scheinen sich in Informationsgesellschaften zu einer „durchlaufenden Kategorie“ (Spinner 2002) entwickelt zu haben. Anders ausgedrückt (Messner 2000: 108):

„Die zunehmende Bedeutung der »funktionalen Institutionen « [gemeint sind Forschung, Beratung und Informationssysteme, KW] reflektiert den Umstand, daß im Verlauf der Entwicklung moderner Gesellschaften neben Macht, Geld und Recht das Wissen zu einem entscheidenden Steuerungsmedium wird.“

Das Zitat drückt aus, dass sich durch diesen Bedeutungsgewinn von Wissen die Grenzen zwischen den staatlichen, gesellschaftlichen und unternehmerischen Sphären zunehmend auflösen würden - ohne Zweifel eine erhebliche Herausforderung für demokratisch verfasste Rechtsstaaten. Aber nicht nur in den genannten Kontexten, sondern in Bezug auf individuelle Handlungszusammenhänge spielen Informationen eine immer wichtigere Rolle - zumindest im Leben jener Menschen, die zu den oben genannten zwei Milliarden

${ }^{3}$ Im Folgenden werden die Ausdrücke „Information“ und „Wissen“ synonym genutzt, obwohl es bspw. aus erkenntnistheoretischer, informationswissenschaftlicher oder informationstheoretischer Sichtweise wesentliche Unterschiede zwischen Informationen und Wissen gibt. Diese verkürzte Sprechweise macht im Kontext der vorliegenden Überlegungen jedoch Sinn, da die erkenntnistheoretischen, informationswissenschaftlichen oder auch informationstheoretischen Aspekte dieser Unterscheidung hier hinter ökonomische Fragen zurücktreten. 
gehören. In rechtsstaatlich verfassten, pluralen und an liberalen Grundsätzen ausgerichteten demokratisch regierten Staaten können Bürger ohne Informationen nicht sinnvoll an in der Regel sehr komplexen politischen Prozessen partizipieren (vgl. z. B. Gradwell 1999; Havick 2000); für Unternehmen mit global verflochtenen Produktionsprozessen, Güterund Finanzströmen, die keine Grenzen mehr kennen, sind Informationen wesentliche Produktionsfaktoren, die über Prosperität oder Untergang dieser Unternehmen und damit letztlich auch über das Gedeihen ganzer Volkswirtschaften mitentscheiden (vgl. Castells 1996; Giesecke 2002: 15); für unseren privaten Lebenswandel kann Nichtinformiertheit ein erhebliches Handicap sein: Ohne adäquate Informationen gehen wir in der Vielfalt unserer Konsumwelt hoffnungslos verloren; ohne Bildung - und damit ohne Zugriff auf Informationen - haben wir auf dem Arbeitsmarkt kaum noch Chancen (vgl. Drake 2001). Informationen stellen also eine wichtige Ressource dar; somit drängt sich die Frage geradezu auf, ob diese Ressource knapp oder gar vollständig verbraucht werden könnte. Wenn diese Frage bejaht werden muss, wäre es an der Zeit, auch in Bezug auf Informationen über Nachhaltigkeit nachzudenken.

Dazu ist es jedoch notwendig zu klären, was sich hinter dem Ausdruck „Information“ verbirgt. Claude E. Shannon und Warren Weaver bspw. betrachteten Information unter mathematisch-statistischen Gesichtspunkten und wollten sich von der Betrachtung semantischer Aspekte lösen (vgl. z. B. Shannon 1998; Weaver 1998). Sie untersuchten Information als Folge von Zeichen, im Extrem als Bitfolgen, und sprachen dabei von Kommunikation, wo in aktuellen Diskussionen in der Regel von Information gesprochen werden würde, da es den beiden um Fragen der Übertragung von Informationen - Kommunikation also - ging und nicht so sehr um deren Speicherung und Verarbeitung. Shannon definierte Information als ein Maß der Unwahrscheinlichkeit des Eintretens eines zufälligen Ereignisses. Weaver war sich allerdings der Verbindungen zwischen der technischen Sicht zu Fragen der Semantik und Pragmatik bewusst; Informationen haben Einfluss auf das menschliche Handeln. Anders ausgedrückt: Informationen sind Ressourcen, die Handlungsmöglichkeiten öffnen und schließen können. Ressourcen wiederum werden z. B. in der politischen Philosophie und in der Ökonomie als Güter bezeichnet, über deren (gerechte) Verteilung nachgedacht werden kann (bspw. Weber 2005). Wenn von Informationen als Gütern gesprochen wird, rückt wiederum der pragmatische Aspekt von Informationen in den Vordergrund: Sie können Bestandteil von Handlungsabfolgen sein und sie beziehen sich auf Tatsachen und Ereignisse in der Welt oder auf andere Informationen wichtig sind also die Inhalte, der Gebrauch, der Nutzen und auch die Konsequenzen von Informationen (Kuhlen 1999: 138/139). Die Einsicht, dass Informationen nicht an sich existieren, sondern immer nur durch ihre Repräsentation als Buchstaben einer Zeitung, als Schallschwingungen oder als digital gespeicherte Daten, verdeutlicht ebenfalls ihren Bezug zu menschlichen Handlungen.

Wenn man nun das bisher Gesagte weiterführte und die verschiedenen Auffassungen von Daten, Informationen und Wissen in den unterschiedlichsten wissenschaftlichen Disziplinen ausführlich berücksichtige, würde dies letztlich nicht zur begrifflichen Klärung beitragen, sondern nur verdeutlichen, dass die Ausdrücke Daten, Informationen und Wissen sehr unscharfe Bedeutungsgrenzen besitzen und disziplinär sehr unterschiedlich verwendet werden; es würde sich zudem zeigen, dass eine Diskussion um die ,richtige“ Definition von Daten, Informationen und Wissen das Verständnis, was Informationsnachhaltigkeit sein könnte, nicht sonderlich fördert. Da Nachhaltigkeit immer etwas mit dem Umgang mit knappen Gütern zu tun hat, macht es deshalb Sinn, sich mit ökonomischen 
Fragen zu beschäftigen und eine Antwort darauf zu geben, welche ökonomischen Eigenschaften Daten, Informationen und Wissen zukommen. Denn dann mag es leichter sein zu verstehen, warum Informationen überhaupt knappe Güter sein können.

\section{KNAPPHEIT UND GÜTERARTEN}

Nach dem bisher Gesagtem könnte der Ausdruck „Informationsnachhaltigkeit“ so verstanden werden, dass Informations- und Kommunikationstechnologie oder auch Informationen selbst dazu genutzt werden, um Nachhaltigkeit im allgemeinen Sinn zu unterstützen; tatsächlich setzen viele Autorinnen und Autoren große Hoffnungen darin, dass IuKTechnologien einen wichtigen Beitrag bei der Gestaltung ressourcenschonender Produktionsweisen leisten könnten (vgl. Caldelli \& Parmigiani 2004; Fuchs 2008). Da die Produktion von IuK-Technologie zudem selbst erhebliche Ressourcen verbraucht, wäre Informationsnachhaltigkeit bspw. im Sinne der Vermeidung von Elektronikschrott, des umweltschonenden Recyclings und ähnlicher Maßnahmen zu verstehen (vgl. Spangenberg 2005). All dies hat viel mit der Idee von Nachhaltigkeit im Sinne der Ressourcenschonung zu tun, doch Informationsnachhaltigkeit in einem eigenständigen Sinn ist damit noch nicht angesprochen.

Der nachhaltige Gebrauch von Rohstoffen betrifft zuvorderst den Wirtschaftskreislauf und den Schutz der Umwelt; damit sind die ökonomische und ökologische Säule der Nachhaltigkeit angesprochen. Zu diesen Säulen kommt die soziale Nachhaltigkeit hinzu: Die sozialen Verhältnisse einer Gesellschaft sollten so gestaltet werden, dass soziale Stabilität, allgemeiner Wohlstand und das friedliche, kooperative und gleichberechtigte $\mathrm{Zu}-$ sammenleben der Bürgerinnen und Bürger einer Gesellschaft auf Dauer und über Generationen gesichert werden können. ${ }^{4}$ Die Einbeziehung der sozialen Säule bei Überlegungen zur Nachhaltigkeit wirft die Frage der Verteilung von Ressourcen auf. Die Zuteilung von Gütern an eine Partei heißt im Fall von materiellen Gütern oft, dass das betreffende Gut einer anderen Partei nicht mehr zur Verfügung steht, dass Zuteilung also Ausschließung bedeuten kann; damit ist eine erste wichtige Eigenschaft materieller Güter angesprochen. Eine weitere wesentliche Eigenschaft ist, ob ein bestimmtes Gut jeweils nur von einer Partei genutzt werden kann und damit Rivalität bezüglich der Nutzung vorliegt. Aufgrund dieser Eigenschaften der Rivalität und Ausschließbarkeit und ihrer Kombinationen ergeben sich vier verschiedene Güterarten: Privatgüter, Club- bzw. Mautgüter, Allmendegüter und schließlich reine öffentliche Güter.

\begin{tabular}{|c|c|c|}
\hline & Ausschließbarkeit & Nicht-Ausschließbarkeit \\
\hline Rivalität & Privatgut (private good) & Allmendegut (common good) \\
\hline $\begin{array}{l}\text { Nicht- } \\
\text { Rivalität }\end{array}$ & Clubgut, Mautgut (club good) & $\begin{array}{l}\text { Reines öffentliches Gut (public } \\
\text { good) }\end{array}$ \\
\hline
\end{tabular}

Tabelle 1: Güterquadrat (vgl. bspw. Ostrom 2005: 24)

Sucht man nach Beispielen für die verschiedenen Güterarten, ist dies für Privat-, Clubbzw. Maut- und Allmendegüter nicht besonders schwer, doch für reine öffentliche Güter

\footnotetext{
${ }^{4}$ Zur Begriffsbildung und zum Umfang von sozialer, ökonomischer und ökologischer Nachhaltigkeit siehe bspw. Dyllick \& Hockerts (2002), Schlossberg \& Zimmermann (2003); auf soziale Nachhaltigkeit in Städten bezogen vgl. z.B. die Beiträge in Polèse \& Stren (2000).
} 
wird man Schwierigkeiten haben, gute Beispiele zu finden. Vor der Entstehung des Umweltschutzgedankens wurde lange gedacht, dass die Natur ein reines öffentliches Gut sei, weil jeder sich nach Belieben aus ihr bedienen könnte, aber immer noch genug davon für die anderen übrig bliebe (Greco \& Floridi 2004: 73):

„For the magnitude of the sea is such, as to be sufficient for the use of all nations, to allow them without inconvenience and prejudice to each other the right of fishing, sailing, or any other advantage which that element affords". Thus Hugo Grotius in 1625. In those days, it seemed that the resources of the oceans were inexhaustible, and hence that regulating their use was unnecessary."

Nachdem alle Kontinente erobert und alle Landstriche in Besitz genommen waren, musste man jedoch erkennen, dass zumindest Land nicht unter die reinen öffentlichen Güter fällt. Entweder man behandelt Land als kollektives Gut und schließt niemand von seiner Nutzung aus; dann aber ist es ein Allmendegut, denn seine Nutzung unterliegt der Rivalität. Oder aber man macht Land zu einem Privatgut und schließt andere von der Nutzung aus. Land in ein Club- oder Mautgut umzuwandeln ist zumindest in der Praxis aufgrund der Rivalität der Nutzung nur sehr bedingt möglich - platt ausgedrückt: auf einem bestimmten Fleckchen Erde kann eben nur ein Mensch stehen. Allmendegüter unterscheiden sich also von reinen öffentlichen Gütern durch die Endlichkeit der jeweiligen Ressource; bekannte Beispiele sind öffentliche Weidegründe (die Allmende) und Hochseefischgründe.

\section{INFORMATIONSGÜTER}

All die genannten Güter stellen materielle Güter dar, so dass die Rivalität der Nutzung oder des Konsums leicht einzusehen ist. Nun scheinen sich Informationen von anderen Ressourcen jedoch deutlich zu unterscheiden. Einmal erzeugt, können Informationsgüter nicht auf die gleiche Weise verbraucht werden wie materielle Güter. Nicht nur können Informationsgüter nicht auf die gleiche Weise verbraucht und damit vermindert werden wie materielle Güter, im Gegenteil: Der Informationsbestand der Welt wächst und wächst. Ging man in Zeiten der Aufklärung noch davon aus, dass es möglich sei, eine vollständige Enzyklopädie des menschlichen Wissens bzw. der den Menschen bekannten Informationen zu erstellen - so Diderot und d'Alembert (1966) mit ihrer „Encyclopédie ou dictionnaire raisonné des sciences, des arts et des métiers“, die sie von 1751 bis 1780 publizierten -, wissen wir heute, dass es wohl kein oberes Limit für die Menge der akkumulierbaren Informationen und damit auch keine abschließende und abgeschlossene Informationssammlung geben wird: der Erzeugung und Sammlung von Informationsgütern scheint keine natürliche Grenze gesetzt zu sein ${ }^{5}$.

Schaut man auf Informationsgüter in Bezug auf die Rivalität der Nutzung, ist auf den ersten Blick nicht zu sehen, dass sie diese Eigenschaft haben: Wenn jemand eine Idee hat und diese einer anderen Person mitteilt, wird die Idee ja nicht weniger; die erste Person kann die Idee nutzen, eine zweite Person ebenso, auch eine dritte und vierte - ad infini-

\footnotetext{
${ }^{5}$ Hierzu gibt es vereinzelt Gegenstimmen: In seinem Buch „Scientific Progress“ von 1978 hat Nicolas Rescher Szenarien beschrieben, die auf ein Ende der Anhäufung neuer Informationen hinauslaufen. In einigen Szenarien wird von Limitierungen des Wissens durch begrenzte (materielle) Ressourcen ausgegangen, in anderen Szenarien von eher prinzipiellen Schranken menschlicher Erkenntnisfähigkeit. Bezüglich der hier verhandelten Fragen besitzen diese Überlegungen aber keine praktische Relevanz und werden deshalb nicht weiter verfolgt.
} 
tum. Dies legt nahe, dass Informationsgüter zumindest von Haus aus nicht der Rivalität des Konsums unterliegen. Doch ganz so einfach ist es nicht, wie ein weiteres Beispiel zeigt: Geheimnisse verlieren ihren Charakter, wenn man sie mit anderen Menschen teilt. Dabei muss der Inhalt des Geheimnisses nicht einmal einen besonderen Wert für andere besitzen - allein die Tatsache, dass andere Menschen das Geheimnis kennen, lässt es verschwinden und somit auch seinen subjektiven Wert. Ein Geheimnis ist daher ein Privatgut, denn es unterliegt der Rivalität und schon per Definition soll es andere vom Gebrauch ausschließen (exkludieren). Geheimnisse sind Informationen bzw. Informationsgüter. Ein Argument gegen die grundsätzliche Annahme, dass Informationsgüter nicht der Rivalität des Konsums und der Ausschließbarkeit unterliegen, ist damit also gefunden.

Dagegen könnte man nun argumentieren, dass ein einziges Gegenbeispiel, das noch dazu wenig mit den hier verhandelten globalen Problemen zu tun hat, sondern allenfalls im privaten Leben einzelner Menschen eine Rolle spielt, kein valides Argument gegen die Annahme der Nichtrivalität von Informationsgütern ist. Selbst wenn man das zugeben möchte, können wir jedoch insbesondere aus der Ökonomie lernen, dass Informationsgüter sehr eng mit Gütern aus der materiellen Welt verbunden sind - und dort herrscht Knappheit und damit Rivalität. Die Produktion von Informationsgütern, bspw. von Musik, Filmen oder auch Computerspielen, ist meist sehr teuer; Produktionskosten von mehreren Hundert Millionen US-Dollar sind für große Hollywoodproduktionen nicht mehr ungewöhnlich. Diese Investitionen müssen irgendwie erwirtschaftet werden, bspw. durch Kinoeintritt, DVD-Verkauf, Verleihgebühren oder Merchandising. Solche Verwertungsweisen bauen darauf auf, dass der jeweilige Rechteinhaber allein kontrollieren kann, unter welchen Bedingungen und damit zu welchem Preis ein Film konsumiert wird. In dem Augenblick, in dem ein Film kopiert und dann über P2P-Netzwerke oder andere Kommunikationskanäle verteilt wird, verliert der Rechteinhaber diese Kontrolle und damit die Möglichkeit, seine Produktionskosten zu erwirtschaften. Damit ist eine Ursache dafür identifiziert, warum Informationsgüter nicht per se als reines öffentliches Gut verstanden werden können: Um die Produktionskosten erwirtschaften zu können, scheint es notwendig, Informationsgüter, wie es bspw. Filme oder Musik sind, als Privatgut, zumindest jedoch als Club- bzw. Mautgut, zu behandeln.

Allgemein gesprochen bedeutet die Umwandlung von Informationsgütern von reinen öffentlichen Gütern zu Privat- oder Club- bzw. Mautgütern eine künstliche Verknappung dieser Güter. Erreicht wird dies durch die Anwendung von Patenten, Urheberrechten und anderen Rechten an Informationsgütern - häufig auch als Immaterialgüter bezeichnet. Wären Informationsgüter nicht durch entsprechende Rechte geschützt, hätte dies, so Dreier \& Nolte (2006: 44)

,[...] zur Folge, dass in öffentliche Güter regelmäßig zu wenig investiert wird. Dadurch kommt es (theoretisch) zu einer strukturellen Unterversorgung der Verbraucher. Eine Lösung für dieses Problem (so genannte »tragedy of the commons «) sind künstliche Anreize zur Investition in die Schaffung und Verbreitung immaterieller Güter durch den Staat. Einen solchen Anreiz bilden die ausschließlichen Rechte des geistigen Eigentums. Durch sie wird die ausschließliche Nutzung, die zunächst faktisch nicht besteht, zumindest mit rechtlichen Mitteln hergestellt.“

Kurzum: Wären Informationsgüter nicht durch Immaterialgüterrechte geschützt, wären sie tatsächlich reine öffentliche Güter, doch brächte dies eben mit sich, dass aufgrund der 
fehlenden ökonomischen Anreize zur Produktion dieser Güter ein Mangel entstehen würde - so die herrschende Meinung in der ökonomischen und juristischen Debatte ${ }^{6}$. Eine völlige Schließung des Zugriffs auf Informationsgüter durch entsprechende Eigentumsrechte, also die Wandlung des reinen öffentlichen Gutes in ein Club- bzw. Mautgut oder gar ein Privatgut, wäre umgekehrt jedoch fatal für soziale Nachhaltigkeit, da Informationsgüter eine enorm wichtige Ressource für Gesellschaften sind.

In diesem Zusammenhang müssen soziale und Informationsnachhaltigkeit in Kombination gebracht werden. In vielen Publikationen, in denen die soziale und ökonomische Stärkung der Entwicklungsländer behandelt wird, ist ein Thema fast immer präsent: Die Versorgung von Individuen, zivilgesellschaftlichen Einrichtungen und Organisationen, Institutionen, der Verwaltung, Politik und Wirtschaft mit Informationen, die für den jeweiligen Handlungsbereich relevant sind (bspw. Arunachalam 1999; vgl. auch Weber 2007 \& 2012). Doch nicht nur auf der gesellschaftlichen Ebene, sondern gleichfalls auf individuellem Niveau, bezogen auf sich entwickelnde Länder ebenso wie auf industrialisierte reiche Staaten, gilt, dass mangelnder Zugriff auf Informationsgüter insbesondere zu Bildungszwecken zu sozialer Exklusion führt (bspw. Pateman 1999). Die Möglichkeit des (lebenslangen) Lernens und die Fähigkeit zum Umgang mit Informationen ebenso wie mit Informations- und Kommunikationstechnologien werden daher als Beitrag zur sozialen Nachhaltigkeit angesehen (vgl. Dudziak 2007; Funk 1999). Der Trend hin zur ökonomischen Schließung des Zugriffs auf Informationsgüter durch die Stärkung bspw. von Urheber- und anderen Immaterialgüterrechten steht dem aber entgegen. Man kann es durchaus als moralische Forderung ansehen, den Zugang zu Informationsgütern so frei wie nur möglich zu gestalten, um gerade den bereits in sozial, ökonomisch und politisch prekären Situationen lebenden Menschen zu helfen und ihnen die Möglichkeit zur Selbsthilfe zu geben (vgl. Britz 2004; Britz \& Lor 2007) - eine Diskussion, die bspw. im Zusammenhang mit Patenten für Medikamente oder Saatgut geführt wird (vgl. Anwander, Bachmann, Rippe \& Schaber 2002).

\footnotetext{
${ }^{6}$ Man könnte einwenden, dass nicht in allen Bereichen der Wissensproduktion im engeren Sinne ökonomische Anreize notwendig sind, damit Wissensbestände erzeugt werden. Da im Folgenden der Fokus auf dem Wissenschaftsbetrieb liegt, ist dieser Einwand bedenkenswert. Man kann ihm jedoch drei Hinweise - Argumente sind das zunächst noch nicht - entgegenstellen. Erstens könnte man bspw. mit Gary S. Becker (1976) die These vertreten, dass menschliches Verhalten grundsätzlich ökonomisch erklärbar ist - auch die Anreizsysteme der Wissenschaft. Zweitens und vermutlich wichtiger kann man darauf hinweisen, dass es hier nicht allein oder nicht einmal primär um Anreize für Personen geht, sondern dass diese in gesellschaftliche (Sub-)Systeme eingebettet sind. Ein zunehmend drittmittelgetriebener Wissenschaftsbetrieb, in dem die staatlichen Zuwendungen gleichermaßen an Verwertbarkeitsforderungen gebunden werden, erhöht den Stellenwert ökonomischer Anreize. Drittens (dies ist aber mehr ein subjektiver Eindruck denn eine empirisch gut belegte Tatsache) kann man gerade in den praxisorientierten Wissenschaftsdisziplinen zunehmend auf Kolleginnen und Kollegen stoßen, die sich nicht nur als Unternehmer verstehen, sondern sich auch so verhalten: Wissenschaft im Sinne Mertons oder Webers ist für diese Personen irrelevant, sie ist stattdessen Mittel zum Zweck des eigenen unternehmerischen Erfolgs und ansonsten ein Kostenfaktor. Die Publikation (und selbst die Produktion) neuen Wissens für die Wissensallmende ist aus dieser Sicht nur insoweit opportun, als dass dies dazu beiträgt, Drittmittel einzuwerben.
} 


\section{DIE TRAGÖDIE DER WISSENSALLMENDE}

Ob nun Filme und Musik zur Wissensallmende gehören, kann man sehr kontrovers diskutieren. Im Folgenden sollen daher nur noch wissenschaftliche Informationsbestände in den Blick genommen werden, denn deren Behandlung ist bereits komplex genug. Selbst hierbei soll noch eine weitere Einschränkung vorgenommen werden, denn die Themenfelder Patente und selbst Urheberrechte werden völlig ausgeklammert; stattdessen soll das wissenschaftliche Publikationswesen als ein wichtiger Teil der Wissensallmende im Vordergrund stehen.

Folgt man Robert K. Merton (1974), so sollen Wissenschaftlerinnen und Wissenschaftler auf eine spezifische Art und Weise desinteressiert an ihrer Arbeit sein. Doch diese Forderung einzuholen ist alles andere als einfach, denn der Wissenschaftsbetrieb ist durch erhebliche Konkurrenz um knappe Ressourcen geprägt. Wer in diesem Betrieb erfolgreich sein will, muss möglichst schnell möglichst viel Reputation erwerben. Das wissenschaftliche Publikationswesen nimmt hinsichtlich dieses Reputationserwerbs nun eine zentrale Stellung ein, denn als Wissenschaftlerin oder Wissenschaftler wird man durch Publikationen sichtbar - in vielen Disziplinen durch die Veröffentlichung von Aufsätzen in renommierten Fachzeitschriften; vor allem in den Geisteswissenschaften nimmt das Buch, wiederum möglichst in einem renommierten Verlag, einen wichtigen Platz ein.

Zeitschriften nutzen heute meist ein Peer-Review-Verfahren, um einerseits die Qualität der veröffentlichten Beiträge zu sichern und andererseits, um der Flut von Einreichungen Herr zu werden. Wer es schafft, durch dieses Sieb hindurchzukommen, gewinnt an Reputation in der jeweiligen scientific community. An diesem System wird nun häufig kritisiert, dass es vor allem Mainstream-Forschung begünstige, also normierenden Charakter habe. Selbst wenn dies nicht (völlig) richtig sein sollte, können sich solche Verfahren dahingehend auswirken, dass wissenschaftliche Arbeiten auf die vermeintlichen oder tatsächlichen Erwartungen der entsprechenden Publikationsmedien hin ausgerichtet werden: Wissenschaftlerinnen und Wissenschaftler sind dann eben nicht mehr auf eine Art und Weise an ihrer Arbeit desinteressiert, wie dies Merton forderte. Dies ist einer jener Mechanismen, die Max Weber (1991) schon 1919 in seinem Aufsatz „Wissenschaft als Beruf" als Konflikt zwischen Wissenschaft als Beruf und als Berufung ansprach.

Dass Wissenschaft nicht in einem luftleeren Raum isoliert von anderen gesellschaftlichen Einflüssen agiert, hat dabei verstärkende Wirkung. In der Wissenschaftssoziologie und in den Wissenschafts- und Technologiestudien (engl.: Science and Technology Studies, STS) wird schon seit geraumer Zeit darauf abgehoben, dass der Wissenschaftsbetrieb, ganz im Gegenteil zu den normativen Ansprüchen Mertons, wesentlich durch Interessenkonflikte und Machtgefälle geprägt sei. Selbst wenn man dies nicht so drastisch sehen möchte, zeigen Studien zum sogenannten Mode 2 der Wissenschaft oder auch zum Triple-Helix-Modell der Wechselwirkung von Wissenschaft, Staat und Wirtschaft (bspw. Etzkowitz \& Leydesdorff 2000; Fujigaki \& Leydesdorff 2000), dass ökonomische Interessen einen hohen Stellenwert im Wissenschaftsbetrieb besitzen. Dies bedroht eine weitere normative Forderung Mertons, den Wissenskommunismus. Damit wird gefordert, dass wissenschaftliche Erkenntnisse mit allen anderen Wissenschaftlerinnen und Wissenschaftlern geteilt werden sollen. Doch Verwertungsinteressen insbesondere von Unternehmen stehen dieser Forderung entgegen. 
Wenn die Wissensallmende umfassend genutzt wird, aber immer weniger Beiträge aus dem Wissenschaftsbetrieb zu ihrem Erhalt geleistet werden, so gefährdet dies den Bestand der Wissensallmende. ${ }^{7}$ Denn im Gegensatz zum klassischen Nachhaltigkeitskonzept, das auf Erhalt eines bestimmten Zustands ausgerichtet ist, muss im Konzept der Informationsnachhaltigkeit stets dafür gesorgt werden, dass neue Informationen und neues Wissen dem bisherigem Bestand hinzugefügt werden: Vor allem Informationen über empirische Sachverhalte veralten; ebenso müssen Theorien und Hypothesen als eine Form des Wissens stets aktualisiert werden, wenn neue empirische Befunde vorliegen, die zur Falsifikation bestehender Annahmen führen; diese neuen Theorien und Hypothesen wiederum erlauben neue Fragen an die Welt und führen zu neuen empirischen Daten - wird dieser Kreislauf durchbrochen, stagniert Wissenschaft und kann dadurch immer schlechter einen Beitrag zur Lösung lebensweltlicher Herausforderungen leisten.

Gleichzeitig jedoch kann der zunehmende Verwertungsdruck, dem der Wissenschaftsbetrieb durch die enge Verflechtung mit Wirtschaft und Staat ausgesetzt ist, die Wissensallmende gefährden. Denn sowohl zur Wissenschaft selbst als auch zur Wissensallemde gehören Karl R. Poppers (1994: 336ff.) „kühne Vermutungen“ (engl.: bold conjectures), die so gar nicht in die Mainstream-Forschung des Wissenschaftsbetriebs hineinpassen. Sie können zu ganz neuen Konzepten führen und sind daher für die Wissenschaft unverzichtbar, doch ihre Generierung ist gefährdet, wenn das Publikationswesen es erschwert, solche Vermutungen zu äußern; ebenso erschwert die Hinwendung der Wissenschaft zur Produktion unmittelbar verwertbarer Erkenntnisse ihre Erzeugung, denn kühne Vermutungen sind in der Regel wirtschaftlich nicht oder zumindest nicht unmittelbar verwertbar ${ }^{8}$.

Daneben, so kann man vermuten, war der Konflikt, der sich durch das Gewinnstreben der großen Wissenschaftsverlage auf der einen und den immer schmaleren Budgets der wissenschaftlichen Bibliotheken auf der anderen Seite ergibt, lange Zeit gar nicht so dramatisch. Kirchgässner (2007: 95/96) schreibt dazu:

,[...] durch das erfolgreiche Bemühen vieler Herausgeber, hochwertige Zeitschriften herauszugeben, wuchs vielen wissenschaftlichen Zeitschriften eine Quasimonopolstellung für ihr Fachgebiet zu [...]. Dies ermöglichte den Verlegern, die Preise dieser Zeitschriften über Jahre hinweg sehr viel stärker anzuheben, als ihre Kostensituation

${ }^{7}$ Im Gegensatz zu materiellen Allmenden geht es im Fall der Wissensallmende nicht um das Problem der Übernutzung. Man kann Wissen im strengen Sinne nicht übernutzen (allerdings fehlnutzen und missbrauchen). Im Gegensatz zu materiellen Gütern verschwindet die Wissensallmende aber auch ohne Nutzung, denn Wissen veraltet (wie gleich ausgeführt). Man kann nicht in einer großen Kraftanstrengung Wissen akkumulieren und dann hoffen, für alle Zukunft gerüstet zu sein. Mit dem vermutlich sehr großen Wissensschatz der antiken Bibliothek von Alexandria wäre uns heute nicht mehr geholfen. Daher sind Anreize zur steten Produktion neuen und sinnvollen Nutzung bestehenden Wissens und der Wissensallmende notwendig.

${ }^{8}$ Dies sollte aber nicht so verstanden werden, dass Verwertbarkeit hier als grundsätzlich von Übel angesehen wird. Ebenfalls sollte die Tatsache, dass heutzutage mithilfe von Evaluationen und ähnlichen Maßnahmen versucht wird, Leistungen im Wissenschaftsbetrieb zu messen und zu bewerten, nicht grundsätzlich verteufelt werden. Denn auch dabei geht es um handfeste Interessen - gerade auch aufseiten der Evaluierten bzw. jener, die sich nicht evaluieren lassen wollen. Eine Hintergrundannahme dieses Textes ist, dass Leistungsmessungen u. Ä. Reaktionen auf Missstände sind (und nicht Auswüchse eines schon begrifflich falsch verstandenen Neoliberalismus), die durch die Erosion dessen zustande kommen, was Helmut F. Spinner (1985) „wissenschaftliches Ethos“ genannt hat. 
dies erfordert hätte. Die Abnehmerseite reagierte relativ unflexibel, denn Wissenschaftler und Bibliothekare konnten erfolgreich ihre Unterhaltsträger bewegen, immer mehr Geld für diese Publikationen bereitzustellen, mit der Begründung, diese Zeitschriften seien für die wissenschaftliche Arbeit unabdingbar. Als Ergebnis können heute einige Großverlage traumhafte Renditen von 24 bis 35 Prozent des Umsatzes einfahren $[\ldots]^{\star 69}$.

Man kann also davon ausgehen, dass zumindest über eine lange Zeit hinweg beide Seiten - die Verlage als auch der Wissenschaftsbetrieb - mit der skizzierten Situation durchaus zufrieden waren. Dies hat sich aber in den letzten Jahren geändert, zumindest zeugen die vielen Open Access-Initiativen davon. Die Euphorie jedoch, die dem Open AccessGedanken zu Anfang entgegengebracht wurde (siehe bspw. die Beiträge in Hess \& Ostrom 2011), scheint inzwischen etwas verebbt zu sein. Es gibt heute zwar eine gar nicht so kleine Zahl von Open Access-Zeitschriften, die ein erhebliches Niveau erreicht haben - in der Regel dadurch, dass sie die gleichen Qualitätssicherungsmaßnahmen wie traditionelle Zeitschriften nutzen, also Peer-Reviewing. Unter diesen Zeitschriften gibt es zudem einige wenige, die ein gewisses Renommee entwickeln konnten, ein Beispiel hierfür sind die Zeitschriften, die im Rahmen der Public Library of Science, kurz: PLOS, erscheinen.

Doch gerade die PLOS-Zeitschriften liefern den Beweis für einen in der Ökonomie recht bekannten Satz: „There is no such thing as a free lunch“ (bspw. Friedman 1975) für die Publikation eines Aufsatzes müssen die Autoren mehrere Tausend US-Dollar bezahlen; andere Verlage mit Open Acess-Geschäftsmodell gehen ähnlich vor. Denn auch das Publizieren mit Open Access verbraucht Ressourcen. Dabei sind die Kosten für die Server, auf denen die Online-Ausgaben von Büchern und/oder Fachzeitschriften liegen, wahrscheinlich noch der kleinste Posten. Doch um bspw. ein Peer-Review durchzuführen, müssen Menschen Zeit aufbringen. Das gilt natürlich auch für traditionelle Zeitschriften und in beiden Fällen bekommen die Reviewer in der Regel keinerlei oder nur eine sehr geringe Vergütung für ihre Arbeit; trotzdem aber fällt diese Arbeit an und muss irgendwie finanziert werden. Das ist nur ein Beispiel für Kosten, die durch das Open AccessKonzept nicht einfach verschwinden. Was verändert wird ist, zu wessen Gunsten diese Kosten aufgebracht werden. Im traditionellen Publikationswesen werden sie privatisiert und tragen zum Gewinn der Verlage bei, im Open Access-Konzept verbleiben sie im Wissenschaftssystem. Das heißt aber erneut, dass die Wissensallmende nicht einfach existiert, sondern aktiv bewirtschaftet werden muss, um weiterhin zur Verfügung zu stehen. Kirchgässner (2007: 95) kommentiert dies lapidar:

„Finanziell betrachtet ist das ganze ein Geschäft zu Lasten Dritter, denn die

Wissenschaftler lassen die Zeitschriften aus dem „Bibliotheksetat“ bezahlen, der in

der Vorstellung der meisten nicht ,ihr“"Etat ist.“

Der Wissenschaftsbetrieb und damit der entsprechende Teil der Wissensallmende sind damit letztlich abhängig davon, dass es jene Dritte gibt, die nicht nur die Forschung selbst, sondern auch das wissenschaftliche Publikationswesen finanzieren. Wie oben schon angedeutet sollte in diesem Zusammenhang von Unternehmensseite nicht allzu viel erhofft werden - ein privatwirtschaftlicher Drittmittelgeber ist an verwertbaren Ergebnissen, nicht

\footnotetext{
${ }^{9}$ Genauere Zahlen kann man bspw. bei Boni (2010: 294) finden.
} 
jedoch an Publikationen in High-Impact-Journalen interessiert und schon gar nicht daran, dass mit den zur Verfügung gestellten Mitteln die Literaturversorgung der akademischen Einrichtungen über den Bedarf für das konkrete Projekt hinaus mitgedeckt wird. Die öffentliche Hand ist hingegen notorisch klamm, von dieser Seite sind kaum mehr Steigerungen für Bibliotheksetats zu erwarten. Doch man kann berechtigt fragen, ob das überhaupt notwendig ist, denn Open Access als Idee stellt schließlich eine richtige und wichtige Frage: Warum müssen Wissenschaftlerinnen und Wissenschaftler (und alle anderen daran interessierten Menschen) für den Zugriff auf Arbeiten, die bereits von der öffentlichen Hand bezahlt wurden, noch einmal teilweise exorbitante Preise bezahlen - das ist in der Tat schwer verständlich. Dazu gleich mehr.

\section{... UND WAS NUN?}

Die Wissensallmende ist einerseits dadurch gefährdet, dass die dort notwendigen Güter durch Immaterialgüterrechte in Privatgüter transformiert werden und dadurch behandelt werden wie materielle Güter; einige Motive, die dazu beitragen, wurden skizziert. Auswüchse dieses Trends lassen sich in Deutschland bspw. bei der Fernleihe von wissenschaftlichen Aufsätzen aus Fachzeitschriften und Sammelbänden beobachten. Hier werden die Immaterialgüterrechte der Urheber bzw. Rechteverwerter überbetont; die Balance zwischen Nutzung und Verwertung ist verloren gegangen. Noch einmal Kirchgässner (2007: 97):

„Kommerzielle Verlage müssen Gewinne machen. Die Frage ist, wieviel Gewinn sie machen. Bei Gewinnmargen um die zehn Prozent - wie sie früher üblich waren - war für jeden Wissenschaftler einsichtig, dass seine Institutionen und Gesellschaften die Zeitschriften auch nicht kostengünstiger bereitstellen können.“

Die lange funktionierende Arbeitsteilung zwischen Wissenschaftsbetrieb und Publikationswesen war ein Geschäft auf Gegenseitigkeit. Sie bot ein funktionierendes Geschäftsmodell für Verlage und Arbeitsentlastung für Wissenschaftlerinnen und Wissenschaftler, denn das Publikationswesen erfüllte wichtige Funktionen für den Wissenschaftsbetrieb und die interne wie externe Wissenschaftskommunikation (vgl. Weber 1999). Doch inzwischen sind mehrere Dinge aus dem Lot geraten: Bei den Verlagen steht zunehmend das Geschäftsmodell bzw. die Gewinnmaximierung im Vordergrund, was nicht nur zu enormen Preissteigerungen bei Zeitschriften geführt hat, sondern auch dazu, dass bei einer Publikation fast alle Arbeitsschritte vergütungsfrei von den Wissenschaftlerinnen und Wissenschaftlern selbst übernommen werden müssen. Daraus zieht Kirchgässner (2007: 97) den Schluss:

„Bei den heute in einigen Bereichen der wissenschaftlichen Zeitschriften realisierten Gewinnmargen der Großverlage zwischen 25 und 35 Prozent, kann sich jeder ausrechnen, dass man mit Herstellungskosten, die um ein Drittel bis um die Hälfte höher liegen als die der Großverlage immer noch ein wirtschaftlich positives Ergebnis erzielen kann.“

Das ist der ökonomische Anlass hinter sehr vielen Open Access-Initiativen. Deren Problem ist - so eine wahrscheinlich ketzerische Hypothese -, dass es so viele sind. Viele der Autorinnen und Autoren in Hess und Ostroms Buch „Understanding Knowledge as a Commons“ (2011) preisen die Tatsache, dass Open Access dezentralisiert funktioniere. Sie setzen große Hoffnungen darauf, dass immer mehr Menschen ihre Texte und andere Informationen frei zugänglich im Internet bereitstellen werden. Das Internet würde dadurch mit der Zeit zu einer gigantischen Bibliothek, die irgendwann das gesamte Weltwissen bereithielte. Das wirft jedoch Fragen auf, denn Open Access macht ja die Funktionen, 
die im Rahmen der Wissenschaftskommunikation übernommen werden, nicht obsolet. Hierzu gehören all die Aufgaben, die bspw. Bibliotheken seit langer Zeit erfüllen und zu den insbesondere die dauerhafte Bereitstellung der Informations- und Wissensbestände sowie deren systematische Erschließung gehören. Bibliotheken erledigen diese Aufgaben nach elaborierten Regelwerken und in einem institutionalisierten Rahmen. Beides ist dringend notwendig auch für Open Source.

Denn Allmenden erfolgreich zu organisieren ohne Institutionen, die Regeln setzen und durchsetzen, funktioniert eigentlich nur in kleinen Gemeinschaften und es müssen dazu einige Bedingungen erfüllt sein (Dietz, Ostrom \& Stern 2003: 1908):

„Effective commons governance is easier to achieve when (i) the resources and use of the resources by humans can be monitored, and the information can be verified and understood at relatively low cost (e.g., trees are easier to monitor than fish, and lakes are easier to monitor than rivers) [...]; (ii) rates of change in resources, resource-user populations, technology, and economic and social conditions are moderate [...]; (iii) communities maintain frequent face-to-face communication and dense social networks - sometimes called social capital - that increase the potential for trust, allow people to express and see emotional reactions to distrust, and lower the cost of monitoring behavior and inducing rule compliance [...]; (iv) outsiders can be excluded at relatively low cost from using the resource (new entrants add to the harvesting pressure and typically lack understanding of the rules); and (v) users support effective monitoring and rule enforcement $[\ldots]$."

Die Wissensallmende, um die es hier geht, stellt jedoch eine Gütersammlung dar, die von Menschen genutzt wird und mit neuen Inhalten beschickt werden soll, die in Bezug darauf eben gerade keiner kleinen Gemeinschaft angehören und die sehr unterschiedliche Interessen haben; es ist unwahrscheinlich, dass sie sich auf gemeinsame Regelwerke bspw. zur Erschließung der Bestände einigen werden. Auch dies bestätigen Dietz, Ostrom \& Stern (2003: 1908):

„Few settings in the world are characterized by all of these conditions. The challenge is to devise institutional arrangements that help to establish such conditions or, as we discuss below, meet the main challenges of governance in the absence of ideal conditions $[\ldots]$. .

Verlässt man sich nun auf eine rein dezentralisierte Wissensallmende, wird es bspw. sehr schwer, die dort verfügbaren Informations- und Wissensbestände zu finden; in diesem Fall fehlten die gerade genannten ,institutional arrangements“. Im Grunde existierten dann zu deren Erschließung nur kommerzielle Suchmaschinen wie Google, deren Betreiberfirmen noch einmal neue, in erster Linie kommerzielle Interessen ins Spiel brächten und allein durch ihre Marktmacht in der Lage wären, die Spielregeln ganz wesentlich mitzubestimmen; ${ }^{10}$ außerdem wirft die technische Implementierung von Suchmaschinen schwerwiegende Fragen bzgl. der korrekten Erschließung von Informationsbeständen auf (vgl. Weber 2011a/b). Sollen solche Abhängigkeiten vermieden werden, gilt es zu begrei-

\footnotetext{
${ }^{10}$ Selbst spezialisierte Suchmaschinen wie Scirus werden von kommerziell ausgerichteten Unternehmen betrieben; Alternativen, wie sie bspw. von Universitäten o. Ä. betrieben werden, erreichen in der Regel nicht die Leistungsfähigkeit großer kommerziell betriebener Suchmaschinen.
} 
fen, dass Gemeinschaften, die an der Erhaltung der Wissensallmende mitarbeiten, faktisch eingebettet sind in größere Zusammenhänge (Berkes 2006: 56): Die Hoffnung darauf, dass allein kleine Gemeinschaften die (globale) Wissensallmende im Sinne der Informationsnachhaltigkeit erhalten und bewirtschaften könnten, wäre schon deshalb verfehlt, weil diese kleinen Gemeinschaften in ein Umfeld eingebettet sind, das sie nicht vollständig kontrollieren können; hier sind viele andere und sehr unterschiedliche Stakeholder beteiligt. Daher ist es nicht mehr die Frage, ob es Institutionen geben muss, die für die Erhaltung und Bewirtschaftung der Wissensallmende Verantwortung tragen, sondern welche (Carroll 2006: 45):

„After the revolutionary euphoria died down, however, many acknowledged that intermediaries are necessary to all kinds of transactions in commerce, culture, and news. Reintermediation soon follows from disintermediation, and the real question the Internet posed was not whether intermediaries are necessary but what kinds of intermediaries are necessary."

Welche Institutionen zum Erhalt und zur Bewirtschaftung der Wissensallmende dazu gehören, lässt sich abschließend nicht beantworten. Sicher werden Archive und Bibliotheken einen wichtigen Platz einnehmen; ebenso Einrichtungen, die Open AccessRepositories betreiben; vermutlich werden Verlage für absehbare Zeit eine wichtige Rolle spielen; Betreiber von Suchmaschinen, ob kommerziell oder nicht, werden für die Erschließung eine kaum zu überschätzende Bedeutung haben. Vor allem aber werden die Produzentinnen und Produzenten der Inhalte der Wissensallmende wichtig sein. Wenn von dort Impulse in Richtung der leichteren Zugänglichkeit der Wissensallmende für alle ausgehen, werden sich die anderen Stakeholder solchen Impulsen nicht verschließen (können). Es gäbe dann die Chance, die (globale) Wissensallmende informationsnachhaltig zu nutzen sowohl für gesellschaftliche wie ökonomische Zwecke, ohne sie zu zerstören $^{11}$.

\section{LITERATUR:}

[1] Anwander, N.; Bachmann, A.; Rippe, Kl. P.; Schaber, P.: Gene patentieren. Eine ethische Analyse. Paderborn: Mentis 2002.

[2] Arunachalam, S.: Information and knowledge in the age of electronic communication: a developing country perspective. In: Journal of Information Science, 25 (1999) 6, S. 465-476.

[3] Becker, G. S.: The economic approach to human behavior. Chicago: University of Chicago Press 1976.

[4] Benkler, Y.; Nissenbaum, H.: Commons-based Peer Production and Virtue. In:

11 Boni (2010: 308f.) nennt Schlussfolgerungen bzgl. der Entwicklung des wissenschaftlichen Publikationswesens, die sich zum Teil mit Aussagen dieses Textes decken, aber auch Aspekte beinhalten, die hier nicht angesprochen werden konnten. Das kann als Hinweis verstanden werden, dass der Erhalt der Wissensallmende vielschichtige Maßnahmen erfordert, die sich nicht in Informationsnachhaltigkeit erschöpfen. Grundsätzlich wird dabei aber auch deutlich, dass Hoffnungen, wie sie bspw. von Benkler \& Nissenbaum (2006: 295) geäußert werden, dass ,socio-technical systems of commons-based peer production offer not only a remarkable medium of production for various kinds of information goods but serve as a context for positive character formation" vielleicht doch etwas zu optimistisch sind, da so viele Stakeholder und damit Interessen eine Rolle bei der Gestaltung der Wissensallemde spielen und spielen müssen; die Hoffnung auf die Formierung besserer Menschen durch die Nutzung des Netzes erscheint in diesem Zusammenhang fast schon etwas naiv. 
Journal of Political Philosophy, 14 (2006) 4, S. 394-419.

[5] Berkes, F.: From community-based resource management to complex systems: the scale issue and marine commons. In: Ecology and Society, 11 (2006) 1, S. 45-59.

[6] Bittlingmayer, U. H.; Bauer, U.: Ungleichheit in der, Wissensgesellschaft'. Zeitdiagnose zwischen naturalisierter Technikentwicklung und invisibilisiertem Klassenkampf. In: Forschungsjournal Neue Soziale Bewegungen, 17 (2004) 2, S. 50-65.

[7] Boni, M.: Analoges Geld für digitale Zeilen: der Publikationsmarkt der Wissenschaft. In: Leviathan, 38 (2010) 3, S. 293-312.

[8] Britz, J. J.: Access to Information: Ethical Guidelines for Meeting the Challenges of the Information Age. In: Pourciau, L. J. (ed.): Ethics and Electronic Information in the Twenty-First Century. West Lafayette/Indiana: Purdue University Press 1999, S. 9-28.

[9] Britz, J. J.: To know or not to know: a moral reflection on information poverty. In: Journal of Information Science, 30 (2004) 3, S. 192-204.

[10]Caldelli, A.; Parmigiani, M. L.: Management Information System? A Tool for Corporate Sustainability. In: Journal of Business Ethics, 55 (2004) 2, S.159-171.

[11]Carroll, M. W.: Creative Commons and the New Intermediaries. In: Michigan State Law Review, 45 (2006), S. 45-65.

[12]Carson, R.: Silent Spring. Boston: Mifflin 1962.

[13]Castells, M.: The Internet Galaxy. Oxford: Oxford University Press 2001.

[14]Castells, M.: The rise of the network society. Malden: Blackwell 1996.

[15] d'Alembert, J. B. le Rond; Diderot, D. (Hrsg.): Encyclopédie ou dictionnaire raisonné des sciences, des arts et des métiers: Nouvelle impression en facsimilé de la première édition de 175-1780. Stuttgart: Friedrich Frommann, 1966.

[16]Dietz, T.; Ostrom, E.; Stern, P. C.: The Struggle to Govern the Commons. In: Science, 302 (2003) 5652, S.1907-1912.

[17]Drake, M. A.: Science, Technology, and Information. In: The Journal of Academic Librarianship, 27 (2001) 4, S. 260-262.

[18]Dreier, Th.; Nolte, G.: Einführung in das Urheberrecht. In: Hofmann, J. (Hrsg.): Wissen und Eigentum. Geschichte, Recht und Ökonomie stoffloser Güter. Bonn: Bundeszentrale für politische Bildung 2006, S. 41-63.

[19]Dudziak, E. A.: Information Literacy and Lifelong Learning in Latin America: the challenge to build social sustainability. In: Information Development, 23 (2007) 1, S. 43-47.

[20]Dyllick, Th.; Hockerts, K.: Beyond the Business Case for Corporate Sustainability. In: Business Strategy and the Environment, 11 (2002), S. 130141.

[21]Etzkowitz, H.; Leydesdorff, L.: The dynamics of innovation: from National Systems and "Mode 2" to a Triple Helix of university-industry-government relations. In: Research Policy, 29 (2000) 2, S.109-123.

[22]Friedman, M.: There's no such thing as a free lunch. La Salle/Illinois: Open Court 1975.

[23]Fuchs, Chr.: The implications of new information and communication 
technologies for sustainability. In: Environment, Development and Sustainability, 10 (2008) 3, S. 291-309.

[24]Fujigaki, Y.; Leydesdorff, L.: Quality control and validation boundaries in a triple helix of university-industry-government: "Mode 2" and the future of university research. In: Social Science Information, 39 (2000) 4, S.635-655.

[25]Funk, K.: Information Networking as an Instrument of Sustainable Development Connectivity, Content, and (Co-) Capacity Building. In: Social Science Computer Review, 17 (1999) 1, S. 107-114.

[26]Gault, F.; McDaniel, S. A.: Continuities and Transformations: Challenges to Capturing Information about the 'Information Society'. In: First Monday, 7 (2002) 2, <http://firstmonday.org/htbin/cgiwrap/bin/ojs/index.php/fm/article/view/928/850 $>$, zuletzt besucht am 21.11.2009.

[27]Germain, Cl. M.: Legal Information Management in a Global and Digital Age: Revolution and Tradition. In: International Journal of Legal Information 35 (2007) 1, S. 134-163,

[28]Giesecke, M.: Von den Mythen der Buchkultur zu den Visionen der Informationsgesellschaft. Frankfurt/Main: Suhrkamp 2002.

[29] Gradwell, J. B.: The Immensity of Technology ... and the Role of the Individual. In: International Journal of Technology and Design Education, 9 (1999), S. 241267.

[30]Greco, G. M.; Floridi, L.: The tragedy of the digital commons. In: Ethics and Information Technology, 6 (2004) 2, S.73-81.

[31]Havick, J.: The impact of the Internet on a television-based Society. In: Technology in Society, 22 (2000), S. 273-287.

[32]Hess, Ch.; Ostrom, E. (eds.): Understanding knowledge as a commons: From theory to practice. Cambridge/Massachusetts: MIT Press 2011 (2007).

[33]Iljon, A.: Library Networks and Electronic Publishing. In: Zeitschrift für Bibliothekswesen und Bibliographie, Sonderheft 65 (1996), Elektronisches Publizieren und Bibliotheken, S. 11-17.

[34]Kirchgäßner, A.: Finanzielle Experimente im wissenschaftlichen Publikationswesen. In: Pipp, E. (Hrsg.): Zugang zum Fachwissen: ODOK ’05. Graz: Wolfgang Neugebauer 2007, S. 92-109.

[35] Kuhlen, R.: Die Konsequenzen von Informationsassistenten. Frankfurt/Main: Suhrkamp 1999.

[36]Kuhlen, R.: Nachhaltigkeit und Inklusivität - Zivilgesellschaft und der Weltgipfel für die Informationsgesellschaft (WSIS). In: Forschungsjournal Neue Soziale Bewegungen, 17 (2004) 2, S. 22-36.

[37]Lor, P. J.; Britz, J. J.: Is a knowledge society possible without freedom of access to information? In: Journal of Information Science, 33 (2007) 4, S. 387-397.

[38]Merton, R. K.: The Normative Structure of Science. In: Merton, R. K. (ed.): The Sociology of Science. Chicago: University of Chicago Press 1974 (1942), S. 267278.

[39]Messner, D.: Gesellschaftliche Determinanten wirtschaftlicher Entwicklung in der Weltmarktwirtschaft. In: Brunkhorst, H.; Kettner, M. (Hrsg.): Globalisierung und Demokratie. Frankfurt/Main: Suhrkamp 2000, S. 90-127.

[40] Ostrom, E.: Understanding Institutional Diversity. Princeton: Princeton 
University Press 2005.

[41]Pateman, J.: Social exclusion: an international perspective on the role of the State, communities and public libraries in tackling social exclusion. In: Journal of Information Science, 25 (1999) 6, S. 445-463.

[42]Polèse, M.; Stren, R. E. (eds.): The social sustainability of cities: diversity and the management of change. Toronto, Buffalo, London: University of Toronto Press 2000.

[43]Popper, K. R.: Objektive Erkenntnis. Hamburg: Hoffmann und Campe, 2. Auflage 1994.

[44]Rai, L. P.; Lal, K.: Indicators of the information revolution. In: Technology in Society, 22 (2000), S. 221-235.

[45]Rescher, N.: Scientific progress. A philosophical essay on the economics of research in natural science. Oxford: Blackwell 1978.

[46] Schlossberg, M.; Zimmerman, A.: Developing Statewide Indices of Environmental, Economic, and Social Sustainability: a look at Oregon and the Oregon Benchmarks. In: Local Environment: The International Journal for Justice and Sustainability, 8 (2003) 6, S. 641-660.

[47]Shannon, Cl. E.: The Mathematical Theory of Communication. In: Shannon, Cl. E.; Weaver, W.: The Mathematical Theory of Communication. Urbana and Chicago: The University of Illinois Press 1998 (1949), S. 29-115.

[48] Spangenberg, J. H.: Will the information society be sustainable? Towards criteria and indicators for a sustainable knowledge society. In: International Journal of Innovation and Sustainable Development, 1 (2005) 1, S.85-102.

[49] Spinner, H. F.: Das „wissenschaftliche Ethos“ als Sonderethik des Wissens: Über das Zusammenwirken von Wissenschaft und Journalismus im gesellschaftlichen Problemlösungsprozess. Tübingen: J.C.B. Mohr 1985.

[50] Spinner, H. F.: Der Karlsruher Ansatz der integrierten Wissensforschung (KAW) im Überblick - Eine kleine Dokumentation. In: Weber, K.; Nagenborg, M.; Spinner, H. F. (Hrsg.): Wissensarten, Wissensordnungen, Wissensregime. Beiträge zum Karlsruher Ansatz der integrierten Wissensforschung. Opladen: Leske+Budrich 2002, S. 249-267.

[51]Weaver, W.: Some Recent Contributions to the Mathematical Theory of Communication. In: Shannon, Cl. E.; Weaver, W.: The Mathematical Theory of Communication. Urbana and Chicago: The University of Illinois Press 1998 (1949), S. 1-28.

[52] Weber, K.: Closing the Digital Divide - lokal, nicht global. In: Bora, A.; Bröchler, St.; Decker, M. (Hrsg.): Technology Assessment in der Weltgesellschaft. Berlin: Edition Sigma 2007, S. 177-188.

[53]Weber, K.: Das Recht auf Informationszugang. Berlin: Frank \& Timme 2005.

[54]Weber, K.: Informationsgerechtigkeit umsetzen. In: Schüller-Zwirlein, A.; Zillien, N. (Hrsg.): Informationsgerechtigkeit. Theorie und Praxis der gesellschaftlichen Informationsversorgung. Berlin: de Gruyter 2012, S. 173-193.

[55]Weber, K.: Search Engine Bias. In: In: Lewandowski, D. (Hrsg.): Handbuch Internet-Suchmaschinen, Band II. Heidelberg: Akademische Verlagsanstalt AKA, 2011, S.265-285. 
[56] Weber, K.: Suchmaschinen und die Repräsentation von Kultur: Zwischen Markt und Gerechtigkeit. In: Banse, G.; Krebs, I. (Hrsg.): Kulturelle Diversität und Neue Medien. Berlin: Trafo, 2011, S. 33-52.

[57]Weber, K.: Wissensarbeitsteilung in der Wissenschaftskommunikation. In: Schulz, W. (Hrsg.): Aspekte und Probleme der didaktischen Wissensstrukturierung. Frankfurt/Main, New York: Peter Lang 1999, S. 149-170.

[58]Weber, M.: Schriften zur Wissenschaftslehre. Stuttgart: Philipp Reclam jun. 1991.

\section{ECONOMIC REFLECTIONS ON THE FUNCTIONING OF THE SCIENCE: THE RIGHTS TO INTANGIBLE PROPERTY, KNOWLEDGE AS A COMMON GOOD AND INFORMATIVE SUSTAINABILITY}

Since the mid-1980s sustainability and sustainable economic activity are both important issues of scholarly as well as public debates. They are discussed particularly in case of the discussions about the consumption of scarce material resources - fossil fuels are paradigmatic examples of such scarce goods as well as examples of resources whose consumption endangers other rare resources like a clean and healthy environment. But since there are not only material rare goods but also intangible goods, it will be analyzed whether concepts like sustainability and sustainable economic activity could be employed with regard to intangible goods like information and knowledge. In order to do that it will be necessary to identify the specific characteristics of information and knowledge. It will be shown that by the application of intellectual property rights these intangible goods can be transformed into scarce resources. If information and knowledge actually must be counted as rare goods it would be reasonable to demand for sustainability with regard to these intangible goods - this shall be called "informational sustainability". If one applies the concept of informational sustainability to the academia concerning the body of knowledge of science one can speak about the knowledge commons. On the one hand the ongoing changes in the academia and the scientific publication system compromise the existence of the knowledge commons; on the other hand one can learn about counter-developments as attempts to maintain the knowledge commons with the idea of Open Access. A detailed analysis shows, however that a romanticizing perspective on the knowledge commons and Open Access as well as a cross-the-board critique of the existing market-driven publication system would be inadequate since both parts of the scientific publication system are depended on each other.

Keywords: sustainability research, resource management, intangible goods, information sustainability, science economics

\section{REFLEKSJE EKONOMICZNE NAD FUNKCJONOWANIEM NAUKI: PRAWA DO DÓBR NIEMATERIALNYCH, WIEDZA JAKO DOBRO WSPÓLNE I INFORMACYJNA ZRÓWNOWAŻONOŚĆ}

Zrównoważony rozwój i zrównoważone gospodarowanie są najpóźniej od połowy lat osiemdziesiątych XX wieku ważnymi koncepcjami zarówno debat naukowych, jak i politycznych. Stają się tematem szczególnie wtedy, kiedy chodzi o postępowanie ze skąpymi zasobami materialnymi - paradygmatycznym przykładem są tutaj kopalne nośniki energii zarówno jako skończone zasoby jak również jako zagrożenie innych skąpych zasobów - takich jak np. czyste środowisko. Ponieważ jednak obok zasobów materialnych 
istnieją również dobra niematerialne, należy zbadać, czy koncepcje zrównoważonego rozwoju i zrównoważonego gospodarowania mogą być wykorzystane także w odniesieniu do dóbr niematerialnych takich jak informacja i wiedza. W tym celu będzie konieczne przede wszystkim opracowanie specyficznych ekonomicznych właściwości informacji i wiedzy. W trakcie takiego opracowywania okazuje się, że dobra niematerialne dzięki zastosowaniu praw do dóbr niematerialnych mogą być uczynione skąpymi zasobami. Jeśli więc również informacje i wiedza faktycznie muszą zostać zaliczone do skąpych zasobów, wówczas także postulowanie zrównoważoności w odniesieniu do dóbr niematerialnych można byłoby określić ją jako „zrównoważonośc informacyjna” - wydaje się mieć sens. Jeśli zastosowuje się koncepcję zrównoważoności informacyjnej do funkcjonowania nauki, wówczas w odniesieniu do zasobów wiedzy naukowej można mówić o wiedzy jako dobru wspólnym. Dokonujące się już od dawna zmiany w funkcjonowaniu nauki i w sferze publikacji naukowych z jednej strony zagrażają zasobom tego wspólnego dobra, z drugiej jednak strony budzą także reakcje przeciwdziałania, które stanowią próby zachowania wspólnotowego charakteru wiedzy na przykład poprzez zapewnianie otwartego dostępu na zasadzie open access. Analiza pokazuje jednak, że zarówno romantyzująca perspektywa wspólnotowości wiedzy i otwartego dostępu, jak i ryczałtowa krytyka pod adresem istniejącego „komercyjnego sprywatyzowania” publikacji naukowych są fałszywe, bo oba segmenty sektora publikacji naukowej są wzajemnie od siebie uzależnione.

Słowa kluczowe: badania dla zrównoważonego rozwoju, zarządzanie zasobami, dobra niematerialne, zrównoważoność informacyjna, ekonomika nauki.

DOI:10.7862/rz.2013.hss.55

Tekst złożono w redakcji: wrzesień 2013.

Przyjęto do druku: marzec 2014. 